\title{
Gut Microbiota Dysbiosis Is Associated with Elevated Bile Acids in Parkinson's Disease
}

\author{
Peipei Li ${ }^{1, *}$, Bryan A. Killinger ${ }^{1,2}$, Elizabeth Ensink ${ }^{1}$, Ian Beddows ${ }^{3}$, Ali Yilmaz ${ }^{4,5}$, Noah Lubben ${ }^{1}$, \\ Jared Lamp ${ }^{6}$ (D), Meghan Schilthuis ${ }^{1}$, Irving E. Vega ${ }^{6}$, Randy Woltjer ${ }^{7}$, J. Andrew Pospisilik ${ }^{3}$, Patrik Brundin ${ }^{1,8}$, \\ Lena Brundin ${ }^{1,8}$, Stewart F. Graham ${ }^{4,5} \mathbb{D}$ and Viviane Labrie ${ }^{1,8}$
}

check for

updates

Citation: Li, P.; Killinger, B.A.; Ensink, E.; Beddows, I.; Yilmaz, A.; Lubben,

N.; Lamp, J.; Schilthuis, M.; Vega, I.E.; Woltjer, R.; et al. Gut Microbiota

Dysbiosis Is Associated with Elevated Bile Acids in Parkinson's Disease.

Metabolites 2021, 11, 29.

https://doi.org/10.3390/

metabo11010029

Received: 26 November 2020 Accepted: 28 December 2020

Published: 4 January 2021

Publisher's Note: MDPI stays neutral with regard to jurisdictional clai$\mathrm{ms}$ in published maps and institutional affiliations.

Copyright: (C) 2021 by the authors. Licensee MDPI, Basel, Switzerland. This article is an open access article distributed under the terms and conditions of the Creative Commons Attribution (CC BY) license (https:// creativecommons.org/licenses/by/ $4.0 /)$.
1 Department of Neurodegenerative Science, Van Andel Institute, Grand Rapids, MI 49503, USA; bryan_killinger@rush.edu (B.A.K.); elizabeth.ensink@vai.org (E.E.); lubbenno@msu.edu (N.L.); mschilthuis@mcw.edu (M.S.); patrik.brundin@vai.org (P.B.); lena.brundin@vai.org (L.B.); viviane.labrie@vai.org (V.L.)

2 Department of Neurological Sciences, Rush University Medical Center, Chicago, IL 60612, USA

3 Department of Epigenetics, Van Andel Institute, Grand Rapids, MI 49503, USA; ian.beddows@vai.org (I.B.); andrew.pospisilik@vai.org (J.A.P.)

4 Metabolomics Department, Beaumont Health, Royal Oak, MI 48073, USA; ali.yilmaz@beaumont.org (A.Y.); Stewart.Graham@beaumont.org (S.F.G.)

5 Department of Obstetrics and Gynecology, Oakland University-William Beaumont School Medicine, Rochester, MI 48309, USA

6 Integrated Mass Spectrometry Unit, Department of Translational Neuroscience, College of Human Medicine, Michigan State University, Grand Rapids, MI 49503, USA; lampjare@mail.msu.edu (J.L.); Irving.Vega@hc.msu.edu (I.E.V.)

7 Department of Pathology, Oregon Health \& Science University, Portland, OR 97239, USA; woltjerr@ohsu.edu

8 Division of Psychiatry and Behavioral Medicine, College of Human Medicine, Michigan State University, Grand Rapids, MI 49503, USA

* Correspondence: lipeipei0611@gmail.com

Abstract: The gut microbiome can impact brain health and is altered in Parkinson's disease (PD) The vermiform appendix is a lymphoid tissue in the cecum implicated in the storage and regulation of the gut microbiota. We sought to determine whether the appendix microbiome is altered in PD and to analyze the biological consequences of the microbial alterations. We investigated the changes in the functional microbiota in the appendix of PD patients relative to controls ( $n=12 \mathrm{PD}, 16 \mathrm{C})$ by metatranscriptomic analysis. We found microbial dysbiosis affecting lipid metabolism, including an upregulation of bacteria responsible for secondary bile acid synthesis. We then quantitatively measure changes in bile acid abundance in PD relative to the controls in the appendix ( $n=15$ PD, $12 \mathrm{C}$ ) and ileum ( $n=20 \mathrm{PD}, 20 \mathrm{C}$ ). Bile acid analysis in the PD appendix reveals an increase in hydrophobic and secondary bile acids, deoxycholic acid (DCA) and lithocholic acid (LCA). Further proteomic and transcriptomic analysis in the appendix and ileum corroborated these findings, highlighting changes in the PD gut that are consistent with a disruption in bile acid control, including alterations in mediators of cholesterol homeostasis and lipid metabolism. Microbially derived toxic bile acids are heightened in PD, which suggests biliary abnormalities may play a role in PD pathogenesis.

Keywords: Parkinson's disease; microbiome; bile acids; appendix; gut

\section{Introduction}

Parkinson's disease (PD) is the second most common neurodegenerative disease, affecting about $1 \%$ of the population over 60 years of age [1]. PD is clinically characterized by motor and non-motor symptoms. Some non-motor features of PD begin many years before the onset of motor symptoms; one of the first symptoms is constipation, pointing toward an early involvement of the gastrointestinal (GI) tract [2]. Aggregated $\alpha$-synuclein ( $\alpha$-syn), a pathological hallmark of PD, is apparent in the GI tract of prodromal PD patients $[3,4]$. 
$\alpha$-syn aggregates in the gut of experimental animal models and has been reported to propagate to the brain and induce nigral neurodegeneration and PD-like motor and non-motor dysfunctions [5,6]. Recently, the appendix has been implicated as one GI tract location that could contribute to PD pathogenesis [7]. The appendix contains an abundance of aggregated $\alpha$-syn, particularly in enteric nerves, with PD patients having higher amounts of these aggregates [7]. The removal of the appendix was associated with a decreased risk for PD in some, but not all, epidemiological studies [7-9]. This suggests that the appendix may be an important tissue to study to advance our understanding of some of the earliest events in this disease.

The appendix is an immunological organ that also acts as a storehouse for the gut microbiota $[10,11]$. The gut microbiota and their metabolites are increasingly being recognized as crucial for brain health [12]. Numerous studies report microbiota changes in the stool of PD patients as compared to healthy controls [13-16]. The appendix contains rich microbial flora, which differ from that of the rectum and stool $[17,18]$. Importantly, the appendix has an anatomically shielded microbiome and can modulate and repopulate the microbiome in the rest of the large intestine $[19,20]$. Consequently, changes in the appendix microbiome may have a widespread effect on the microbiota of the intestine, which may be reflected in changes in stool microbiota. Furthermore, inflammation in the periphery and the brain has been proposed to have a central role in PD, and the microbiome and the host immune system have a bidirectional effectual relationship [12,21,22]. Microbiota can modify inflammatory responses and immunity in the gut, and the lymphoid tissue of the appendix is particularly relevant since it is especially rich in lymphocytes compared to the rest of the GI tract [11]; microbial metabolites have direct access to the immune cells within the lymphoid follicles of the appendix [23-25]. Thus, the dysregulation of the appendix microbiota may be involved in PD, but this has yet to be examined.

One important function of the gut microbiota is their involvement in the biotransformation of bile acids. Bile acids aid in the absorption of dietary lipids and affect glucose homeostasis, inflammation, gastrointestinal functions, as well as blood-brain barrier integrity and signaling in the brain [26-28]. In the liver, primary bile acids are synthesized from cholesterol. After being released from the gall bladder, most primary bile acids are reabsorbed in the ileum for transport back to liver [26]. The remaining primary bile acids that enter the large intestine are converted by the microbiota (largely by those in Clostridium clusters XIVa and XI) into secondary bile acids-deoxycholic acid (DCA), lithocholic acid (LCA), and ursodeoxycholic acid (UDCA) [26]. Lithocholic acid and DCA are hydrophobic bile acids that are cytotoxic at elevated physiological concentrations [29,30]. Increases in LCA and DCA have been implicated in intestinal inflammation, liver injury, cholestasis, and gallstone formation $[29,31-33]$. Whether there are hydrophobic bile acid changes that impact PD risk is unknown.

To test our hypothesis that the appendix will show microbiota changes in PD, with an impact on the associated transcription and protein homeostasis in the gut, we performed a multi-omic analysis of the appendix microbiome in PD patients and controls. We performed a multi-omic analysis of the appendix microbiome, including metabolomics, proteomics, and transcriptomics in PD patients and controls.

\section{Results}

\subsection{Microbiota Changes in PD Appendix}

Here, we performed a comprehensive microbiome analysis in the appendix tissue of PD and controls ( $n=12$ and 16 respectively; Supplementary File 1$)$ using metatranscriptomic sequencing, which profiles the functionally active microbiota. We had on average $14,288,947 \pm 5,008,507$ reads per sample, and found transcripts for 65 genera, 37 families, 20 orders, 15 classes, and 9 phyla. We did not find changes in the richness of microbiota communities between PD and controls at any taxonomic level (alpha diversity; Figure S1). The appendix of PD and controls also had a similar overall microbial community composition (beta-diversity; Figure S1). The most abundant bacteria in the appendix overall were 
Lachnospiraceae, Ruminococcaceae, Porphyromonadaceae, Enterobacteriaceae, and Bacteroidaceae, together accounting for $68.6 \%$ of the relative family abundance (Figure S1). We also compared the appendix microbial community identified to that found in surgically isolated, healthy appendix tissues from a previous study and found significant correlation (order level: $\mathrm{R}=0.91, p<10^{-14}$; family level: $\mathrm{R}=0.26, p<0.05$; Pearson's correlation).

In an analysis examining the abundance of microbial taxa, differences between the appendix microbiota of PD and controls were observed at all taxonomic levels $(q<0.05$; Figure 1a; Supplementary File 2). The most significant change in the PD appendix microbiota relative to controls was in the order of Clostridiales, particularly an increase in Peptostreptococcaceae and Lachnospiraceae. The appendix of PD patients also had a prominent increase in Burkholderiales and decrease in Methanobacteriales. Furthermore, the PD appendix had decreases in genera Odoribacter, Clostridium, unclassified Sutterellaceae, and Escherichia.

a

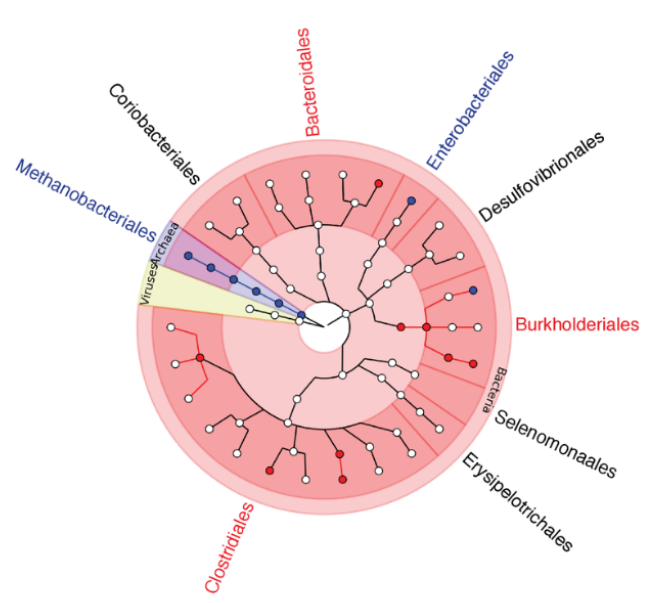

b

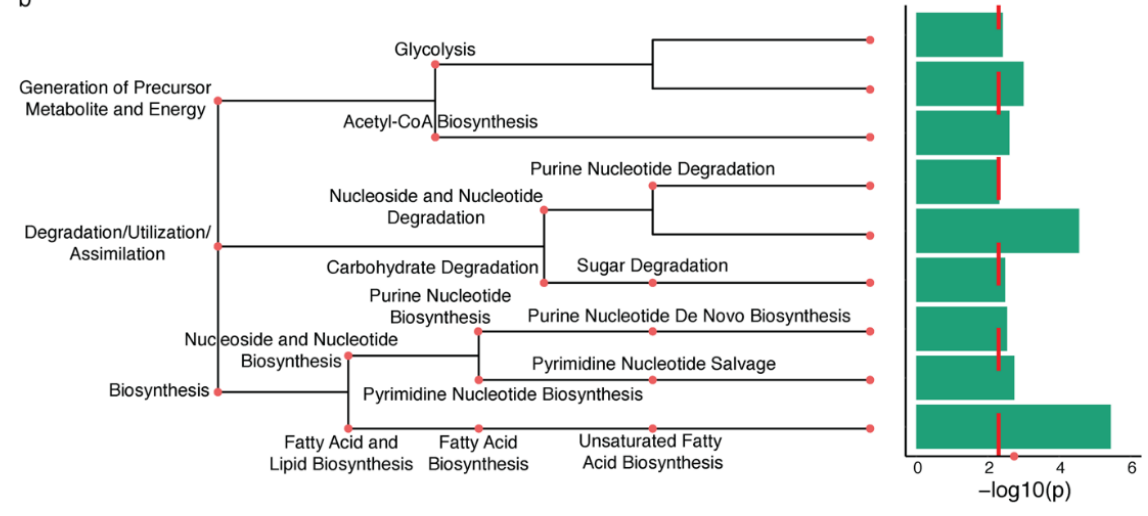

Figure 1. The Parkinson's disease (PD) appendix exhibits shifts in the active microbiota that affect lipid metabolism. Metatranscriptomic analysis was used to determine the changes in the functional microbiota in the PD appendix ( $n=12 \mathrm{PD}, 16$ controls). (a) Microbiota changes in the PD appendix. Metatranscriptome data were analyzed by MetaPhlAn2 and a zero-inflated Gaussian mixture model in metagenomeSeq, adjusting for age, sex, RNA integrity number (RIN), and post-mortem interval. Results are displayed using GraPhlAn, showing the taxonomic tree with the kingdom in the center, and branching outwards to phylum, class, order, family, and genus. Microbial taxa highlighted in red are increased in PD, and blue are decreased in $\mathrm{PD}(q<0.05$, metagenomeSeq). (b) Microbiota metabolic processes are altered in the PD appendix. Top microbial pathways are altered in PD as identified by HumanN2. Red dashed line denotes $q<0.1$ pathways as determined by metagenomeSeq. 


\subsection{Proteomic Changes in the PD Gut}

Then, we examined the major microbial metabolic pathways altered in the PD appendix. We report that the most significant microbial pathway change was a loss of fatty acid metabolism and the dysregulation of the lipid metabolism $(q<0.1$; Figure 1b; Supplementary File 2). Considering the prominent disruption of the lipid metabolic pathways in the microbiome of PD patients, we analyzed the human proteome in the appendix tissue of PD and controls. Pathway analysis of the proteomic data highlighted a reduction in proteins affecting lipid metabolism $(q<0.05$; Figure 2a; Supplementary File 3). There was also a dysregulation of pathways involved in protein localization, antigen presentation, glycolysis, and immune activity in the PD appendix (Figure 2a). Overall, the proteomic changes affecting lipid homeostasis in the PD appendix corroborate the microbial pathway alterations affecting lipids in patients.

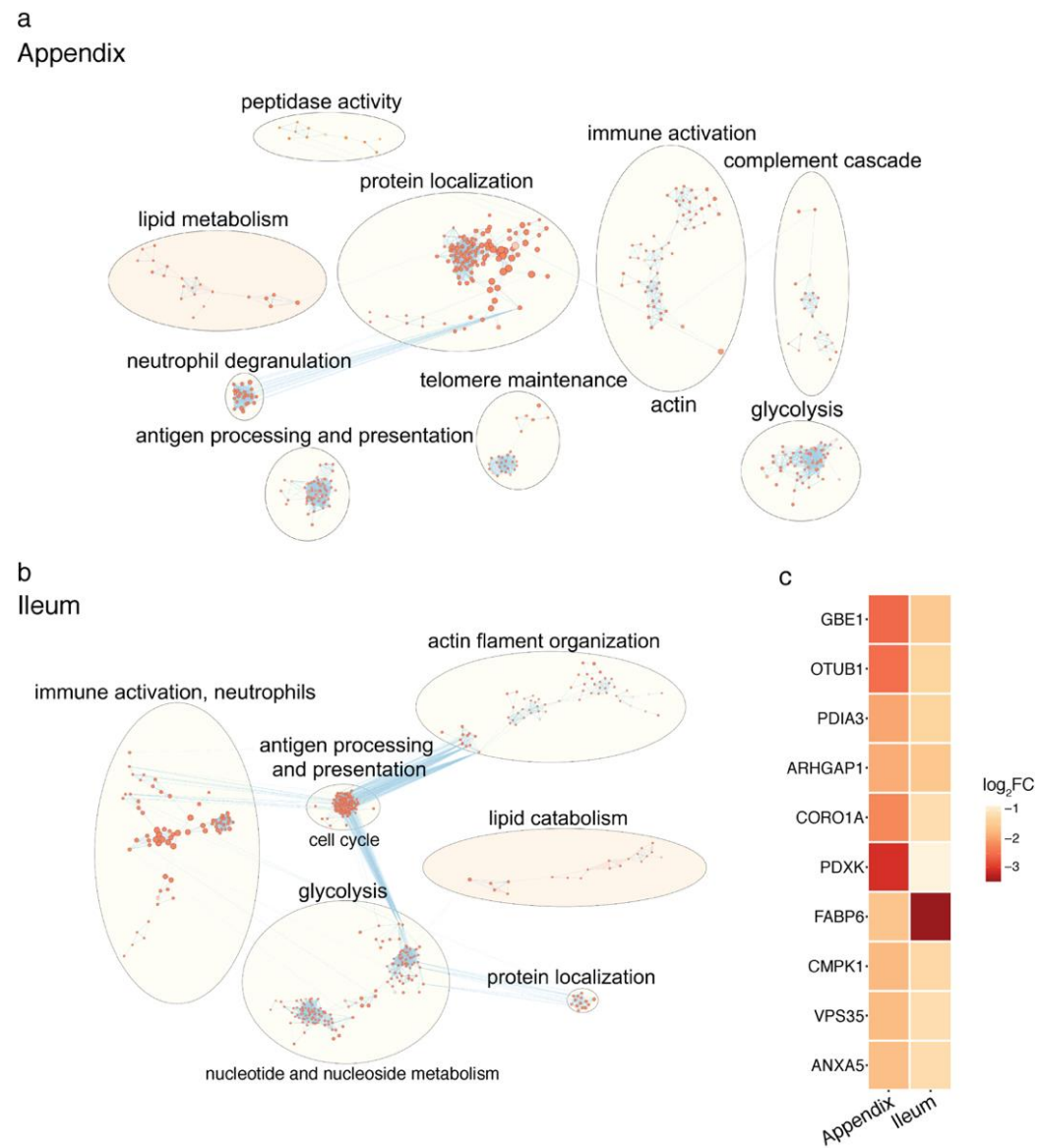

Figure 2. Proteomic analysis identifies the altered lipid metabolism pathways in the PD appendix and ileum. Pathway enrichment analysis of proteomic changes in the PD appendix relative to controls ( $n=3$ PD, 3 controls) (a) and in PD ileum relative to controls ( $n=4$ PD, 4 controls) (b). Pathway analysis of quantitative proteomic data was performed using g:Profiler. Nodes are pathways altered in the PD appendix that were clustered into functionally similar networks by EnrichmentMap (nodes are $q<0.05$ pathways, hypergeometric test). Node size represents the number of genes in the pathway gene set, and the edges connect pathways with similar gene sets ( 0.7 similarity cutoff). The lipid metabolism pathway network is highlighted in peach. (c) Top 10 proteins that were most consistently altered in the PD appendix and ileum. Heatmap showing the proteins ranked as the most consistently disrupted in the PD appendix and ileum, as determined by a robust ranking algorithm. Log fold change is shown, and red signifies greater disruption in PD. 
In addition, we profiled the proteome of the ileum of PD patients and compared it to that of controls with the aim of identifying significantly altered metabolic pathways. We found that the PD ileum had a decrease in lipid metabolism, as observed in the PD appendix $(q<0.05$; Figure 2b). In the PD ileum, there was also a dysregulation of antigen processing and presentation, immune activation, glycolysis, and actin filament organization $(q<0.05$; Figure $2 b)$. We then determined the proteins most consistently altered in the PD ileum and appendix [34]. We found that both the PD ileum and appendix had a strong decrease in fatty acid binding protein 6 (FABP6), the intracellular bile acid transporter involved in returning bile acids to enterohepatic circulation (Figure 2c).

\subsection{Microbiota-Driven Bile Acid Changes in the Gut of PD Patients}

In addition to identifying perturbations in the lipid metabolism in the PD appendix, we also report changes in microbiota responsible for generating hydrophobic secondary bile acids (Clostridium cluster XI and Burkholderiales). Consequently, we measured the bile acid levels in the PD appendix. We accurately quantified 15 bile acids in PD and healthy controls ( $n=15$ and 12, respectively) (Figure 3a; Supplementary File 4) and found an 18.7-fold increase in LCA and a 5.6-fold increase in DCA in the PD appendix relative to the controls ( $p<0.05$; Figure 3; Supplementary File 4). We did not observe any changes in primary bile acids or total bile acid levels in the PD appendix, but elevated concentrations of secondary bile acids produced by the microbiota $(p<0.05)$. In addition, we measured the concentrations of bile acids in the ileum of PD patients ( $n=20$ PD and 20 controls) and once again report no changes in the primary bile acid concentrations but do show a significantly marked increase in LCA in the PD ileum as compared to the controls (3.6-fold; Figure 3; Supplementary File 4).

a

\section{- Proinflammatory $\uparrow P D$ vs $C$ appendix $\uparrow P D$ vs $C$ ileum}

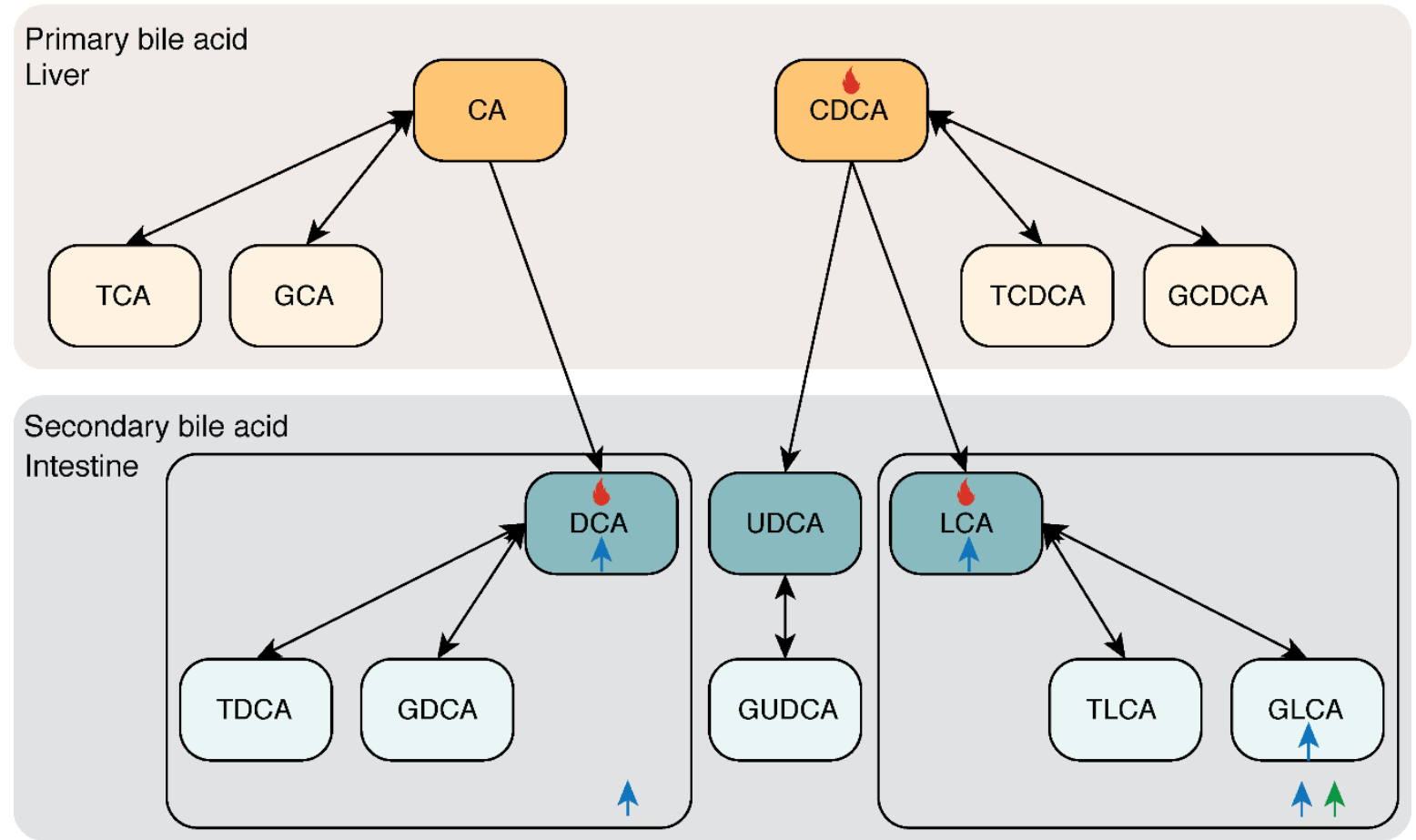

Figure 3. Cont. 
b
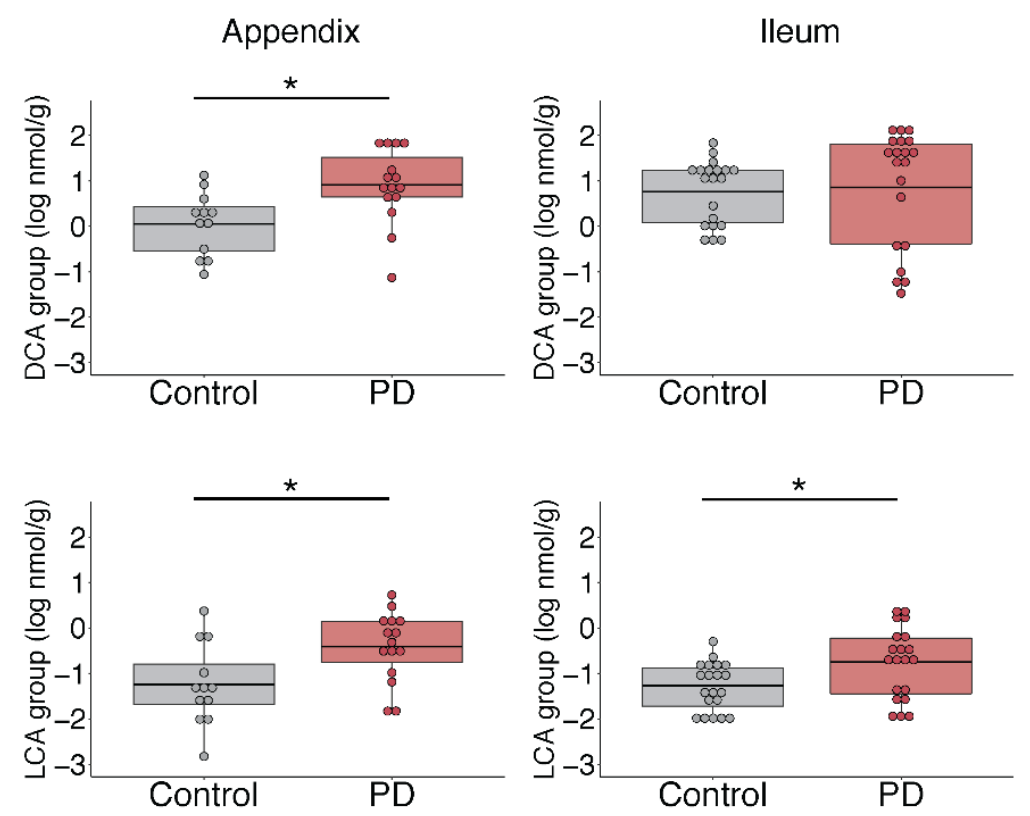

Figure 3. Increase in the microbiota-derived secondary bile acids in the appendix of PD patients. Bile acid analysis was performed by liquid chromatography-mass spectrometry in the PD and control appendix ( $n=15$ PD, 12 controls) and ileum ( $n=20 \mathrm{PD}, 20$ controls). Bile acid changes were determined by robust linear regression, controlled for age, sex, and postmortem interval. (a) Illustration of the bile acid changes identified in this study and the bile acid pathway. Primary bile acids are generated in the liver and secondary bile acids are produced by microbiota in the intestine. In the secondary bile acid section of the image, boxes highlight the DCA and LCA groups (DCA, LCA and their respective conjugates). Bile acids increased in the PD appendix or PD ileum, relative to controls, are marked by a blue and green arrow, respectively. The flame symbol denotes hydrophobic bile acids that have proinflammatory effects when elevated. (b) Secondary bile acid changes in the PD appendix and PD ileum. The boxplot center line represents the mean, the lower and upper limits are the first and third quartiles (25th and 75th percentiles), and the whiskers are $1.5 \times$ the interquartile range. ${ }^{*} p<0.05$, robust linear regression.

\subsection{Bile-Associated Transcriptomic Changes in the PD Gut}

Since we found an abundance of microbiota-derived bile acids in PD, we investigated whether PD patients had a differential expression of genes important for bile acid biosynthesis, signaling, and transport. In the ileum of PD and controls, we examined the transcript levels of nuclear receptors that regulate bile acid and cholesterol homeostasis (FXR and LXR), bile acid-sensing receptor (TGR5), transporters for bile reabsorption (ASBT, OST $\alpha / O S T \beta, F A B P 6$ ), and transporters for cholesterol reabsorption and efflux (NPC1L1 and $A B C G 5 / A B C G 8$, respectively). We also examined the liver tissue of PD and controls for transcript levels of these genes or their liver-specific functional equivalents (NTCP and FABP1), as well as enzymes for bile acid biosynthesis in the liver (CYP7A1 and CYP27A1). We found that the PD ileum had significantly elevated levels of gene transcripts involved in cholesterol homeostasis and transport $(p<0.05$; Figure 4a; Supplementary File 5). In the liver, we did not observe changes in bile acid-related transcripts (Figure $4 b$; Supplementary File 5). In conclusion, the human transcriptomic and proteomic changes in the PD gut are consistent with a disruption in bile acid control, including alterations in mediators of cholesterol homeostasis and lipid metabolism. 
a

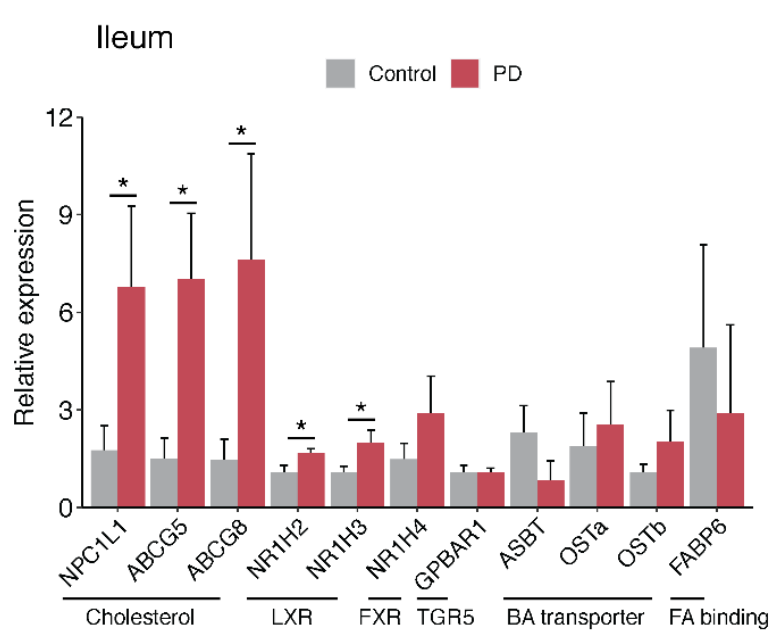

b

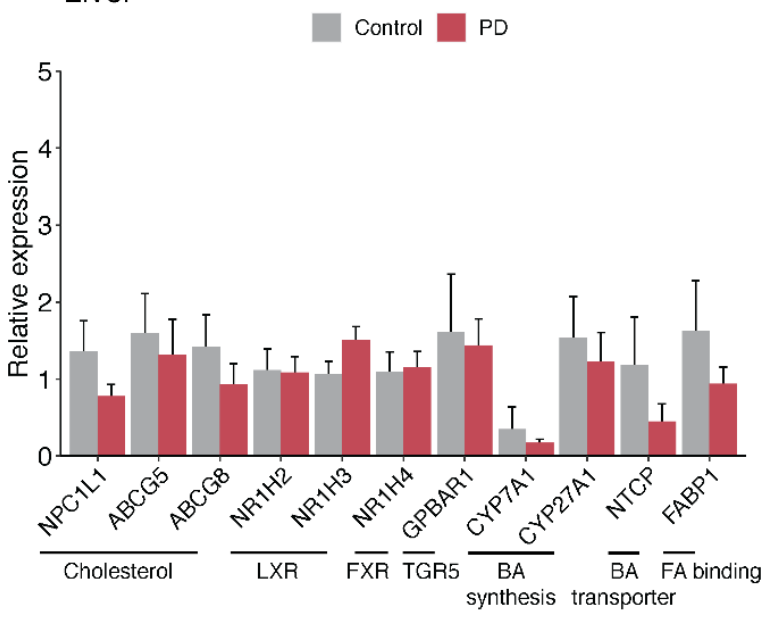

Figure 4. Dysfunctional cholesterol and lipid metabolism in the PD ileum. Transcript levels of genes in the ileum (a) and liver (b) affecting the abundance of cholesterol and bile in the enterohepatic circulation. Transcript levels of genes affecting cholesterol and bile acid homeostasis $(N R 1 H 2, N R 1 H 3, N R 1 H 4, G P B A R 1)$ and their transport and reabsorption into the enterohepatic circulation (NPC1L1, ABCG5, ABCG8, ASBT, OST $\alpha$, OST $\beta, F A B P 6)$ were examined in the ileum. In the liver, the transcript levels of these genes or the equivalent bile acid transporters (NTCP, FABP1) were examined, as well as the rate-limiting enzymes for bile acid production (CYP7A1, CYP27A1). Transcript levels were analyzed by qPCR and normalized to housekeeping genes (villin1, $\beta$-actin). The relative expression \pm s.e.m in the ileum $(n=8$ PD, 6 controls) and liver ( $n=6$ PD, 6 controls). ${ }^{*} p<0.05$, one-way ANOVA.

\section{Discussion}

Our metatranscriptomic analysis revealed significant differences in the appendix microbiota of PD patients, which are closely related to bile acid dysregulation in the gut. The microbial taxa and pathway alterations we identified led us to further hypothesize that bile acid metabolism might be directly associated with the etiopathogenesis of PD. Thus, we investigated the potential changes in bile acid metabolism in PD using metabolomics, proteomics, and transcriptomics. We report significant increases in the concentrations of secondary bile acids in the human appendix and ileum $(p<0.05)$. Furthermore, proteomic and transcriptomic analysis support the dysregulation of lipid metabolism and cholesterol homeostasis in the gut of PD patients.

We found significant microbiota differences in the PD appendix using a metatranscriptomic approach. Unlike the prior $16 \mathrm{~S}$ rRNA and metagenomic studies of the PD microbiome, our metatranscriptomic study has the advantage of capturing only active species. The appendix microbial community identified was similar to that found in surgically isolated, healthy appendix tissues in a previous study, demonstrating reliability in our methods [17]. Another strength of our study is the unique location of the survey; microbiota shifts in the appendix are particularly relevant given the role of the appendix in affecting the microbiota in other intestinal regions [11]. Differences between the appendix and fecal microbiomes limit comparison with previous studies of the PD microbiota; however, an increase in Clostridiales cluster XI has been observed in PD stool previously [35]. Nonetheless, one limitation of our study is that it does not accurately distinguish microbiota changes which may contribute to the causes of PD from those that are consequences of the disease. Both constipation (a common symptom of PD) and PD medication may influence the gut microbiota [35]. Though we cannot exclude constipation as a contributing factor, it is unlikely to explain all the changes observed, since increases in both LCA and DCA increase colonic peristalsis which decreases the fecal transit time [36-38]. Although we controlled for age and sex, additional clinical variables such as medication or the duration 
of disease were not available for this dataset but would be valuable to test for correlations; we aim to consider this in future work.

The microbiome clearly plays a role in the health of the nervous system [12,39,40], and our results implicate bile acid metabolism as another pathway by which changes in the microbiota may contribute to the etiology and pathogenesis of PD. Bacterial species that are responsible for the production of secondary bile acids in the large intestine were elevated in the PD appendix. We report significant increases in Burkholderiales which is a bacterial genus with a broad environmental distribution [41]. Burkholderia can cause severe inflammation in immunocompromised individuals [41] and produce kynurenine and quinolinate [42], which are proinflammatory metabolites associated with symptom severity in PD [43]. Burkholderia has also been reported to infect the brain [44,45]. Of particular interest, Burkholderia species encode the rate-limiting enzyme for secondary bile acid synthesis (bile-acid dehydratase) [46], highlighting the fact that the appendix microbiome of PD sufferers has an enrichment of microbiota that metabolize bile acids.

In line with the microbiota shifts as observed in the PD appendix, we found an increase in the secondary bile acids LCA and DCA in the ileum of PD patients. LCA and DCA are highly hydrophobic, and their increase can have pro-inflammatory and direct cytotoxic effects $[27,29,30]$. These effects could propel the accumulation of pathological $\alpha$-syn aggregates, which could potentially propagate from the gut to the brain through retrograde transport $[47,48]$. Changes in the appendix are especially relevant since the appendix holds an abundance of $\alpha$-syn even in healthy individuals [11]. Alterations in the microbiome have been correlated with changes in melatonin synthesis along with serum DCA levels [49]; melatonin, which is highly produced in the appendix, can suppress the accumulation of toxic $\alpha$-syn and may contribute to PD etiopathogenesis. Thus, local melatonin levels in the PD appendix may impact both the levels of secondary bile acids and $\alpha$-syn aggregation [50]. In addition, since bile acids can modulate the immune response [32,33,51,52], LCA and DCA may contribute to T cell activation, which has been implicated in PD pathogenesis [53], and/or the microbial-driven bile acid changes may be a response to $\alpha$-syn aggregation and concurrent inflammation in the gut of PD sufferers. Further in vivo models are essential to elucidate the effects of secondary bile acid metabolism on PD pathology.

Our study provides some insight into the potential causes behind the changes in the microbiota and bile acid composition in PD, which merit further investigation. In the PD patients, there was no evidence of an overproduction of primary bile acids: primary bile acids and the total bile acid pool size in the PD ileum remained similar to the controls, and we did not detect transcript abnormalities in enzymes responsible for primary bile acid synthesis in the PD liver. However, PD patients may have impaired bile acid reuptake in the ileum, as indicated by the prominent decrease in FABP6, a protein responsible for the efficient transport of primary bile acids through enterocytes for recirculation [54]. Thus, disrupted bile acid transport in the ileum and increased bile-metabolizing bacteria in the large bowel combined could be responsible for the elevated levels of secondary bile acids as observed in PD. These disruptions may also contribute to the observed abnormalities in cholesterol homeostasis in the PD gut, as demonstrated by the transcriptional increase in cholesterol transporters and the proteomic disruption of lipid metabolism pathways. It is also worth noting that changes in bile acids can in turn modulate the composition of the microbiota. Though this study does not delineate which changes occur first in PD, the bidirectional relationship between the microbiota and bile acid composition creates a system which, once triggered, may lead to a self-reinforced condition of dysbiosis, peripheral inflammation, and $\alpha$-syn aggregation.

Targeting the appendix microbiome and bile acids may be an innovative approach for future therapeutics. Our results support microbiome transplantation as a potential treatment for PD [55]. In addition, preventing the damaging effects of LCA and DCA with the hydrophilic, anti-inflammatory bile acid UDCA could benefit PD patients. UDCA has been shown to counteract the effects of hydrophobic secondary bile acids in the liver and 
gallbladder [56,57]. UDCA also has neuroprotective effects [58] and is currently being tested in clinical trials for PD [59].

In sum, our findings provide a novel look into the appendix microbiota in PD and demonstrate microbially mediated bile acid disturbances (Figures S2 and S3). Though further investigation is needed, bile acids could play a key role at the intersection of microbiome dysbiosis, inflammation, and $\alpha$-syn misfolding. Considering the relative accessibility of GI the tract and existing therapies for bile acid-related disorders, targeting microbial-derived secondary bile acids may be a new avenue for the earlier diagnosis and alleviation of PD symptoms.

\section{Materials and Methods}

\subsection{Human Tissue Samples}

The human appendix, ileum, and liver tissue from PD patients and controls was obtained from the Oregon Brain Bank with information on demographics (age, sex), tissue quality (postmortem interval), and pathological staging for each individual (Supplementary File 1). Tissue was collected and flash frozen within an average postmortem interval of $14 \mathrm{~h}$ and shipped frozen on dry ice. PD cases were selected based on pathologically confirmed presence of brain Lewy body pathology and the loss of midbrain neurons, and control individuals were selected for the absence of such pathology. Cases and controls were balanced by sex and age across groups. All human postmortem tissue work had approval from the Van Andel Institute ethics committee (IRB \#15025).

\subsection{Metatranscriptomic Analysis of PD Appendix Microbiota}

To profile the functional microbiome changes in the PD appendix, we performed a metatranscriptomic analysis of the appendix of 12 PD and 16 controls. Frozen appendix tissue ( $20 \mathrm{mg}$ ) was homogenized using a Covaris cryoPREP pulverizer and then in $1 \mathrm{~mL}$ of TRIzol (Life Technologies, Carlsbad, CA, USA) with a ceramic bead-based homogenizer (Precellys, Bertin Instruments, Montigny-le-Bretonneux, France). Total RNA was isolated according to the TRIzol manufacturer's instructions, treated with RNase-free DNase I (Qiagen, Hilden, Germany) at room temperature for $30 \mathrm{~min}$, and cleaned up with the RNeasy Mini Kit (Qiagen). Total RNA yield and quality was determined using a NanoDrop ND-1000 (Thermo Fisher Scientific, Waltham, MA, USA) and an Agilent Bioanalyzer 2100 system (Agilent Technologies, Santa Clara, CA, USA). Libraries were prepared by the Van Andel Genomics Core from 300 ng of total RNA using the KAPA RNA HyperPrep Kit with RiboseErase (v1.16; Kapa Biosystems, Wilmington, MA, USA). RNA was sheared to 300-400 bp. Prior to PCR amplification, cDNA fragments were ligated to NEXTflex dual adapters (Bioo Scientific, Austin, TX, USA). The quality and quantity of the finished libraries were assessed using a combination of Agilent DNA High Sensitivity chip (Agilent Technologies, Inc.), QuantiFluor dsDNA System (Promega Corp., Madison, WI, USA), and Kapa Illumina Library Quantification qPCR assays (Kapa Biosystems, Wilmington, MA, USA). Individually indexed libraries were pooled, and $100 \mathrm{bp}$, single-end sequencing was performed on an Illumina NovaSeq6000 sequencer using an S1 100 cycle kit (Illumina Inc., San Diego, CA, USA), with all libraries run on a single lane to return an average depth of 37 million reads per library. Base calling was done by Illumina RTA3, and the output of NCS was demultiplexed and converted to a FastQ format with Illumina Bcl2fastq v1.9.0.

The preprocessing of metatranscriptomic data involved the removal of sequencing adapters and low-quality bases from sequencing reads using Trim Galore (v0.5.0). The transcriptomic data were aligned to human genome (GRCh38/hg38) with the twopassMode basic algorithm in STAR (v2.5.2b) [60]. Reads that did not align to the human genome (STAR option outReadsUnmapped) were then input to MetaPhlAn2 (v2.7.7) [61], which gives kingdom to species-level resolution (db_v20). We then performed the functional profiling of the microbial community for the same non-human reads using HUMAnN2 (v0.11.1) [62] with the UniRef 90 database. Pathway abundance data were normalized to counts per million using the inbuilt HUMAnN2 functionality. To test for differential taxa abundance 
between the PD and control, proportional microbial compositional data from MetaPhlAn2 were imported into R (v3.6) and converted back to counts for all taxa-level ids (features). To perform statistical analysis, we used the cumulative sum scaling normalization and the zero-inflated Gaussian mixture model from the bioconductor package metagenomeSeq (v1.28.0) [63]. Feature and pathway abundance data were examined using the fitZig function to determine the microorganisms and pathways related to PD, adjusting for age, sex, postmortem interval, and RIN. $p$-values were derived from the empirical Bayes moderated F-statistic and adjusted for multiple testing correction using the BenjaminiHochberg method, with $q<0.05$ set as the threshold for statistical significance.

\subsection{Mass Spectrometry and Proteomics Analysis}

Here, quantitative proteomic analysis was performed to determine the host biological pathways altered in the PD appendix and ileum. For this analysis, we used existing proteomic data from the PD and control appendix ( $n=3$ individuals/group; PXD015079) and generated new proteomic data for the PD and control ileum ( $n=4$ individuals/group). Mass spectrometry for the appendix and ileum samples was performed using the same protocol by the Integrated Mass Spectrometry Unit at Michigan State University. The wet tissue weight of each sample ( $30 \mathrm{mg}$ tissue/sample) was measured and a 5-fold lysate buffer (20 mM Tris Base (pH 7.4), $150 \mathrm{mM} \mathrm{NaCl}, 1 \mathrm{mM}$ EGTA, $1 \mathrm{mM}$ EDTA, $5 \mathrm{mM}$ sodium pyrophosphate, $30 \mathrm{mM}$ NaF, 1X Halt Protease Inhibitor Cocktail (Thermo Fisher Scientific) was used to homogenize the tissue on ice with a tissue grinder (Tissue Master 125, Omni International). The homogenate was centrifuged at $18,407 \times g$ for $10 \mathrm{~min}$ at $4{ }^{\circ} \mathrm{C}$ and the supernatant was retained. Protein concentration in each sample was determined using a BCA assay (Pierce BCA Protein Assay, Thermo Fisher Scientific). Protein lysates (10 $\mu \mathrm{g}$ ) were denatured using $25 \mathrm{mM}$ ammonium bicarbonate/ $80 \%$ acetonitrile and incubated at $37^{\circ} \mathrm{C}$ for $3 \mathrm{~h}$. The samples were dried and reconstituted in $50 \mu \mathrm{L}$ of $25 \mathrm{mM}$ ammonium bicarbonate/50\% acetonitrile/trypsin/LysC solution (1:10 and 1:20 w/w trypsin:protein and LysC:protein, respectively) and digested overnight at $37^{\circ} \mathrm{C}$. The samples were dried and reconstituted in $50 \mu \mathrm{L}$ of $25 \mathrm{mM}$ ammonium bicarbonate $/ 5 \%$ acetonitrile.

Samples were loaded onto an UltiMate 3000 UHPLC system with online desalting. Each sample $(10 \mu \mathrm{L})$ was separated using a C18 EASY-Spray column $(2 \mu \mathrm{m}$ particles, $25 \mathrm{~cm} \times 75 \mu \mathrm{m}$ ID) and eluted using a $2 \mathrm{~h}$ acetonitrile gradient into a Q-Exactive HF-X mass spectrometer. Data-dependent acquisition for the full MS was set using the parameters described in the supplementary methods. Each sample was run in triplicate. The mass spectra from each technical replicate were searched against the Uniprot human database (filtered proteome_3AUP000005650) using the Label-free quantification (LFQ) method in Proteome Discoverer (v. 2.2.0.388, 2017). Data-dependent acquisition for the full MS was set using the following parameters: resolution 60,000 (at $200 \mathrm{~m} / \mathrm{z}$ ), Automatic gain control (AGC) target $3 \times 10^{6}$, maximum Injection time (IT) $45 \mathrm{~s}$, scan range 300 to 1500 $m / z$, dynamic exclusion $30 \mathrm{~s}$. Fragment ion analysis was set with the following parameters: resolution 30,000 (at $200 \mathrm{~m} / z$ ), AGC target $1 \times 10^{5}$, maximum IT $100 \mathrm{~ms}$, TopN 20, isolation window $1.3 \mathrm{~m} / z$, Normalized collision energy (NCE) at 28. Each sample was run in triplicate. The mass spectra from each technical replicate were searched against the Uniprot human database (filtered-proteome_3AUP000005650) using the LFQ method in Proteome Discoverer (v. 2.2.0.388, 2017) set as follows: at least 2 peptides (minimum length $=6$, minimum precursor mass $=350 \mathrm{Da}$, maximum precursor mass $5000 \mathrm{Da}$ ), tolerance set to $10 \mathrm{ppm}$ for precursor ions and 0.02 Da for fragment ions ( $\mathrm{b}$ and y ions only), dynamic modification was set for methionine oxidation $(+15.995 \mathrm{Da})$ and N-terminus acetylation (+42.011 Da), target FDR (strict minimum value 0.01$)$, Delta Cn minimum value 0.05$)$. LFQ was calculated using the following parameters: the ratio calculation was set to pairwise ratio based, maximum allowed fold change 100, ANOVA (background based).

The technical replicates from each biological sample were pooled to perform diagnosis comparisons, using a non-nested test. Proteins were quantified using the pairwise peptide ratio information from extracted peptide ion intensities. Only proteins with abundances 
recorded in at least $50 \%$ of samples were considered. Proteins with a log fold change between groups exceeding \pm 0.2 were considered altered. The pathway analysis of proteins altered in the PD appendix and ileum was performed using g:Profiler [64], with networks determined by EnrichmentMap and clustered by AutoAnnotate in Cytoscape (v3.7.1) [65] To identify the proteins that were most altered, we first determined the proteins that exhibited significant changes and had the same direction of change in both the PD appendix and ileum. These altered proteins were ranked by log fold change, ranking separately for appendix and ileum. We then determined the proteins most consistently altered using the aggregateRanks function from the RobustRankAggreg package (v1.1) [34].

\subsection{Bile Acid Sample Preparation and Metabolite Quantification}

Liquid chromatography-mass spectrometry (LC-MS) was used to measure the primary and secondary bile acids in the appendix and ileum of PD patients and controls (appendix, $n=12$ controls, 15 PD; ileum, $n=20$ controls, 20 PD). Tissues (25 mg) were homogenized using a bead homogenizer at $5500 \mathrm{rpm}$ for $30 \mathrm{~s}$ in the $300 \mu \mathrm{L}$ of extraction solvent ( $85 \%$ ethanol and $15 \%$ phosphate-buffered saline solution). Samples were then sonicated at $4{ }^{\circ} \mathrm{C}$ for $10 \mathrm{~min}$. Proteins and other impurities were removed by centrifugation at $13,000 \times g$ for $30 \mathrm{~min}$ at $4^{\circ} \mathrm{C}$. The supernatant was collected and $10 \mu \mathrm{L}$ was loaded onto the Biocrates Bile Acid kit (Biocrates Life Sciences). Data were acquired using an Acquity I-class UPLC (Waters) coupled with a Xevo TQ-S mass spectrometer (Waters). All specimens were acquired in accordance with the protocol for the Biocrates Bile Acids kit. Bile acid concentrations (nmol per gram of tissue weight) were calculated utilizing the Biocrates MetIDQ software and TargetLynx (Waters). For the group-level analyses, the concentration of the bile acid and its glycine and taurine conjugates were summed. For example, the LCA group was the sum of LCA, GLCA, and TLCA, and the DCA group was the sum of DCA, GDCA and TDCA. For the all-primary, all-secondary, and total bile acid analyses, the respective bile acids and their conjugates were summed.

Bile acid data were normalized by $\log 10$ transformation, as previously described [66]. Bile acid changes in the PD appendix and ileum were determined by multivariate robust linear regression models with empirical Bayes from the limma (v3.30.13) statistical package [67], adjusting for age, sex, and postmortem interval.

\section{5. qPCR Analysis of Gene Transcripts Involved in Bile Acid and Cholesterol Homeostasis}

We examined the transcriptional changes of genes involved in bile acid transport and cholesterol homeostasis in the PD ileum $(n=6$ controls, 8 PD) and liver $(n=6$ controls, 6 PD). Samples from the PD and healthy controls were matched for sex, age, and postmortem interval. Tissue (30-50 mg per sample) was homogenized in $1 \mathrm{~mL}$ TRIzol with a handheld homogenizer (Biospec) for the rileum and with Precellys bead tubes (Bertin Corp, Rockville, MD, USA) for the liver. Following standard TRIzol RNA extraction, samples were treated with DNase (Qiagen) for $30 \mathrm{~min}$. RNA cleanup was performed using a RNeasy column (Qiagen) according to the manufacturer's instructions, with the addition of two $75 \%$ ethanol washes. Isolated RNA quantity was determined with a NanoDrop 2000 spectrophotometer, and RNA integrity was confirmed with an Agilent 2100 Bioanalyzer. RNA was converted to cDNA using a High Capacity cDNA kit (Applied Biosystems, Carlsbad, CA, USA). Samples were analyzed by qPCR with TaqMan reagents (Applied Biosystems; Supplementary File 6), using $25 \mathrm{ng}$ of cDNA per qPCR reaction. Samples were run in triplicate and the results were normalized to plate standardization controls. The delta delta CT values of gene transcripts were used to determine the statistical changes in the ileum and liver of PD relative to the controls, normalized to housekeeping control genes ( $\beta$-actin and HPRT for the liver, $\beta$-actin or villin for ileum). Statistical analysis was performed using one-way ANOVA with $p<0.05$ considered to be significant changes. 
Supplementary Materials: The following are available online at https:/ / www.mdpi.com/2218-1 989/11/1/29/s1, Figure S1: Microbial diversity in the human appendix; Figure S2: Experimental design and main findings of our study investigating functional changes in the appendix microbiota in PD; Figure S3: Graphical summary of microbiota and biliary changes in PD; Supplementary File 1: Demographic and clinical information for human samples; Supplementary File 2: Differences in PD appendix microbiota and related pathways identified by metatranscriptomic analysis; Supplementary File 3: Proteomic changes in the human PD appendix and ileum; Supplementary File 4: Changes in bile acid composition in the human PD appendix, and ileum and in the cecal patch of mice with synucleinopathy and gut inflammation; Supplementary File 5: Differences in gene transcripts related to bile acid homeostasis in the human PD ileum and liver identified by qPCR; Supplementary File 6: Primers used in liver and ileum qPCR experiments.

Author Contributions: Conceptualization, P.L., B.A.K., E.E. and V.L.; data curation, B.A.K., E.E., A.Y., N.L., J.L., M.S., I.E.V. and S.F.G.; formal analysis, P.L. and I.B.; funding acquisition, J.A.P., P.B., L.B. and V.L.; investigation, P.B., L.B., S.F.G. and V.L.; methodology, P.L. and V.L.; project administration, V.L.; resources, R.W.; supervision, J.A.P., P.B., L.B., S.F.G. and V.L.; visualization, P.L.; writing-original draft, P.L., E.E. and V.L.; writing-review and editing, P.L., E.E., R.W., P.B., L.B. and S.F.G. All authors have read and agreed to the published version of the manuscript.

Funding: This work was supported by a Farmer Family Foundation grant award to P.B., with L.B., J.A.P., and V.L. as co-investigators. V.L. is supported by grants from the Department of Defense (W81XWH1810512), the National Institute of Neurological Disorders and Stroke (1R21NS11261401, 1R01NS113894-01A1, 1R01NS114409-01A1), and a Gibby \& Friends vs. Parky Award. S.F.G. is supported by grants from the National Institute of Neurological Disorders and Stroke (1R01NS11083801A1), the National Institutes on Aging (1R21AG067083-01), the Alzheimer's Association (AARG-17530020) and the Michael J. Fox Foundation (MJFF16201).

Institutional Review Board Statement: The study was conducted according to the guidelines of the Declaration of Helsinki, and approved by the ethics committee of the Van Andel Research Institute (IRB \#15025; Sep. 2018).

Informed Consent Statement: Informed consent was obtained from all subjects involved in the study.

Data Availability Statement: All sequencing data used in this study are available from the NCBI Gene Expression Omnibus (GEO) database under the accession number GSE135743. The mass spectrometry proteomics data of human ileum has been deposited to the ProteomeXchange Consortium via the PRIDE partner repository with the dataset identifier PXD020988. The human appendix proteomic data was obtained from PXD015079.

Acknowledgments: We dedicate this paper in memorial of our research adviser, mentor, and friend, Viviane Labrie. We thank the Van Andel Institute Genomics and Bioinformatics and Biostatistics Cores and Michigan State University Genomics Core. We thank the Oregon Brain Bank, for the tissue provided.

Conflicts of Interest: P.B. has received commercial support as a consultant from Axial Biotherapeutics, Calico, CuraSen, Fujifilm-Cellular Dynamics International, IOS Press Partners, LifeSci Capital LLC, Lundbeck A/S, Idorsia and Living Cell Technologies LTD. He has received commercial support for grants/research from Lundbeck A/S and Roche. He has ownership interests in Acousort AB and Axial Biotherapeutics and is on the steering committee of the NILO-PD trial. S.G. receives commercial support as a consultant from Coleman Research and Biogen. No other authors have conflicts of interest.

\section{References}

1. Ascherio, A.; Schwarzschild, M.A. The epidemiology of Parkinson's disease: Risk factors and prevention. Lancet Neurol. 2016, 15, 1257-1272. [CrossRef]

2. Kalia, L.V.; Lang, A.E. Parkinson's disease. Lancet 2015, 386, 896-912. [CrossRef]

3. Stokholm, M.G.; Danielsen, E.H.; Hamilton-Dutoit, S.J.; Borghammer, P. Pathological $\alpha$-synuclein in gastrointestinal tissues from prodromal Parkinson disease patients. Ann. Neurol. 2016, 79, 940-949. [CrossRef]

4. Hilton, D.; Stephens, M.; Kirk, L.; Edwards, P.; Potter, R.; Zajicek, J.; Broughton, E.; Hagan, H.; Carroll, C. Accumulation of $\alpha$-synuclein in the bowel of patients in the pre-clinical phase of Parkinson's disease. Acta Neuropathol. 2014, 127, $235-241$. [CrossRef] 
5. Kim, S.; Kwon, S.-H.; Kam, T.-I.; Panicker, N.; Karuppagounder, S.S.; Lee, S.; Lee, J.H.; Kim, W.R.; Kook, M.; Foss, C.A.; et al. Transneuronal Propagation of Pathologic $\alpha$-Synuclein from the Gut to the Brain Models Parkinson's Disease. Neuron 2019, 103, 627-641.e627. [CrossRef]

6. Van Den Berge, N.; Ferreira, N.; Gram, H.; Mikkelsen, T.W.; Alstrup, A.K.O.; Casadei, N.; Tsung-Pin, P.; Riess, O.; Nyengaard, J.R.; Tamgüney, G.; et al. Evidence for bidirectional and trans-synaptic parasympathetic and sympathetic propagation of alpha-synuclein in rats. Acta Neuropathol. 2019, 138, 535-550. [CrossRef]

7. Killinger, B.A.; Madaj, Z.; Sikora, J.W.; Rey, N.; Haas, A.J.; Vepa, Y.; Lindqvist, D.; Chen, H.; Thomas, P.M.; Brundin, P.; et al. The vermiform appendix impacts the risk of developing Parkinson's disease. Sci. Transl. Med. 2018, 10. [CrossRef]

8. Marras, C.; Lang, A.E.; Austin, P.C.; Lau, C.; Urbach, D.R. Appendectomy in mid and later life and risk of Parkinson's disease: A population-based study. Mov. Disord. 2016, 31, 1243-1247. [CrossRef]

9. Liu, B.; Fang, F.; Ye, W.; Wirdefeldt, K. Appendectomy, Tonsillectomy and Parkinson's Disease Risk: A Swedish Register-Based Study. Front. Neurol. 2020, 11, 510. [CrossRef]

10. Donaldson, G.P.; Lee, S.M.; Mazmanian, S.K. Gut biogeography of the bacterial microbiota. Nat. Rev. Microbiol. 2016, 14, 20-32. [CrossRef]

11. Killinger, B.; Labrie, V. The Appendix in Parkinson's Disease: From Vestigial Remnant to Vital Organ? J. Parkinson's Dis. 2019, 9, S345-S358. [CrossRef]

12. Fung, T.C.; Olson, C.A.; Hsiao, E.Y. Interactions between the microbiota, immune and nervous systems in health and disease. Nat. Neurosci. 2017, 20, 145-155. [CrossRef] [PubMed]

13. Scheperjans, F.; Aho, V.; Pereira, P.A.B.; Koskinen, K.; Paulin, L.; Pekkonen, E.; Haapaniemi, E.; Kaakkola, S.; Eerola-Rautio, J.; Pohja, M.; et al. Gut microbiota are related to Parkinson's disease and clinical phenotype. Mov. Disord. 2015, 30, 350-358. [CrossRef] [PubMed]

14. Bedarf, J.R.; Hildebrand, F.; Coelho, L.P.; Sunagawa, S.; Bahram, M.; Goeser, F.; Bork, P.; Wüllner, U. Functional implications of microbial and viral gut metagenome changes in early stage L-DOPA-naïve Parkinson's disease patients. Genome Med. 2017, 9, 39. [CrossRef] [PubMed]

15. Perez-Pardo, P.; Dodiya, H.B.; Engen, P.A.; Forsyth, C.B.; Huschens, A.M.; Shaikh, M.; Voigt, R.M.; Naqib, A.; Green, S.J.; Kordower, J.H.; et al. Role of TLR4 in the gut-brain axis in Parkinson's disease: A translational study from men to mice. Gut 2019, 68, 829-843. [CrossRef]

16. Heinzel, S.; Aho, V.T.E.; Suenkel, U.; von Thaler, A.K.; Schulte, C.; Deuschle, C.; Paulin, L.; Hantunen, S.; Brockmann, K.; Eschweiler, G.W.; et al. Gut Microbiome Signatures of Risk and Prodromal Markers of Parkinson Disease. Ann. Neurol. 2020, 88, 320-331. [CrossRef]

17. Jackson, H.T.; Mongodin, E.F.; Davenport, K.P.; Fraser, C.M.; Sandler, A.D.; Zeichner, S.L. Culture-independent evaluation of the appendix and rectum microbiomes in children with and without appendicitis. PLoS ONE 2014, 9, e95414. [CrossRef]

18. Tytgat, H.L.P.; Nobrega, F.L.; van der Oost, J.; de Vos, W.M. Bowel Biofilms: Tipping Points between a Healthy and Compromised Gut? Trends Microbiol. 2019, 27, 17-25. [CrossRef]

19. Masahata, K.; Umemoto, E.; Kayama, H.; Kotani, M.; Nakamura, S.; Kurakawa, T.; Kikuta, J.; Gotoh, K.; Motooka, D.; Sato, S.; et al. Generation of colonic IgA-secreting cells in the caecal patch. Nat. Commun. 2014, 5, 3704. [CrossRef]

20. Sanchez-Alcoholado, L.; Fernandez-Garcia, J.C.; Gutierrez-Repiso, C.; Bernal-Lopez, M.R.; Ocana-Wilhelmi, L.; Garcia-Fuentes, E.; Moreno-Indias, I.; Tinahones, F.J. Incidental Prophylactic Appendectomy Is Associated with a Profound Microbial Dysbiosis in the Long-Term. Microorganisms 2020, 8, 609. [CrossRef]

21. Johnson, M.E.; Stecher, B.; Labrie, V.; Brundin, L.; Brundin, P. Triggers, Facilitators, and Aggravators: Redefining Parkinson's Disease Pathogenesis. Trends Neurosci. 2019, 42, 4-13. [CrossRef] [PubMed]

22. van Kessel, S.P.; El Aidy, S. Bacterial Metabolites Mirror Altered Gut Microbiota Composition in Patients with Parkinson's Disease. J. Parkinsons Dis. 2019, 9, S359-S370. [CrossRef] [PubMed]

23. Schulthess, J.; Pandey, S.; Capitani, M.; Rue-Albrecht, K.C.; Arnold, I.; Franchini, F.; Chomka, A.; Ilott, N.E.; Johnston, D.G.W.; Pires, E.; et al. The Short Chain Fatty Acid Butyrate Imprints an Antimicrobial Program in Macrophages. Immunity 2019, 50, 432-445.e437. [CrossRef] [PubMed]

24. Bachem, A.; Makhlouf, C.; Binger, K.J.; de Souza, D.P.; Tull, D.; Hochheiser, K.; Whitney, P.G.; Fernandez-Ruiz, D.; Dahling, S.; Kastenmuller, W.; et al. Microbiota-Derived Short-Chain Fatty Acids Promote the Memory Potential of Antigen-Activated CD8(+) T Cells. Immunity 2019, 51, 285-297.e285. [CrossRef] [PubMed]

25. Chun, E.; Lavoie, S.; Fonseca-Pereira, D.; Bae, S.; Michaud, M.; Hoveyda, H.R.; Fraser, G.L.; Gallini Comeau, C.A.; Glickman, J.N.; Fuller, M.H.; et al. Metabolite-Sensing Receptor Ffar2 Regulates Colonic Group 3 Innate Lymphoid Cells and Gut Immunity. Immunity 2019, 51, 871-884.e876. [CrossRef] [PubMed]

26. Wahlström, A.; Sayin, S.I.; Marschall, H.-U.; Bäckhed, F. Intestinal Crosstalk between Bile Acids and Microbiota and Its Impact on Host Metabolism. Cell Metabolism. 2016, 24, 41-50. [CrossRef]

27. Hegyi, P.; Maléth, J.; Walters, J.R.; Hofmann, A.F.; Keely, S.J. Guts and Gall: Bile Acids in Regulation of Intestinal Epithelial Function in Health and Disease. Physiol. Rev. 2018, 98, 1983-2023. [CrossRef]

28. McMillin, M.; DeMorrow, S. Effects of bile acids on neurological function and disease. FASEB J. 2016, 30, 3658-3668. [CrossRef]

29. Chen, M.L.; Takeda, K.; Sundrud, M.S. Emerging roles of bile acids in mucosal immunity and inflammation. Mucosal Immunol 2019, 12, 851-861. [CrossRef] 
30. Pavlidis, P.; Powell, N.; Vincent, R.P.; Ehrlich, D.; Bjarnason, I.; Hayee, B. Systematic review: Bile acids and intestinal inflammationluminal aggressors or regulators of mucosal defence? Aliment. Pharmacol. Ther. 2015, 42, 802-817. [CrossRef]

31. Berr, F.; Mayer, M.; Sackmann, M.F.; Sauerbruch, T.; Holl, J.; Paumgartner, G. Pathogenic factors in early recurrence of cholesterol gallstones. Gastroenterology 1994, 106, 215-224. [CrossRef]

32. Hang, S.; Paik, D.; Yao, L.; Kim, E.; Trinath, J.; Lu, J.; Ha, S.; Nelson, B.N.; Kelly, S.P.; Wu, L.; et al. Bile acid metabolites control TH17 and Treg cell differentiation. Nature 2019, 576, 143-148. [CrossRef] [PubMed]

33. Song, X.; Sun, X.; Oh, S.F.; Wu, M.; Zhang, Y.; Zheng, W.; Geva-Zatorsky, N.; Jupp, R.; Mathis, D.; Benoist, C.; et al. Microbial bile acid metabolites modulate gut ROR $\gamma+$ regulatory T cell homeostasis. Nature 2020, 577, 410-415. [CrossRef] [PubMed]

34. Kolde, R.; Laur, S.; Adler, P.; Vilo, J. Robust rank aggregation for gene list integration and meta-analysis. Bioinformatics 2012, 28, 573-580. [CrossRef] [PubMed]

35. Weis, S.; Schwiertz, A.; Unger, M.M.; Becker, A.; Faßbender, K.; Ratering, S.; Kohl, M.; Schnell, S.; Schäfer, K.-H.; Egert, M. Effect of Parkinson's disease and related medications on the composition of the fecal bacterial microbiota. NPJ Parkinson's Dis. 2019, 5, 28. [CrossRef] [PubMed]

36. Alemi, F.; Poole, D.P.; Chiu, J.; Schoonjans, K.; Cattaruzza, F.; Grider, J.R.; Bunnett, N.W.; Corvera, C.U. The receptor TGR5 mediates the prokinetic actions of intestinal bile acids and is required for normal defecation in mice. Gastroenterology 2013, 144, 145-154. [CrossRef]

37. Misawa, N.; Higurashi, T.; Takatsu, T.; Iwaki, M.; Kobayashi, T.; Yoshihara, T.; Ashikari, K.; Kessoku, T.; Fuyuki, A.; Matsuura, T.; et al. The benefit of elobixibat in chronic constipation is associated with faecal deoxycholic acid but not effects of altered microbiota. Aliment. Pharmacol. Ther. 2020, 52, 821-828. [CrossRef]

38. Ge, X.; Zhao, W.; Ding, C.; Tian, H.; Xu, L.; Wang, H.; Ni, L.; Jiang, J.; Gong, J.; Zhu, W.; et al. Potential role of fecal microbiota from patients with slow transit constipation in the regulation of gastrointestinal motility. Sci. Rep. 2017, 7, 441. [CrossRef]

39. Erny, D.; Hrabě de Angelis, A.L.; Jaitin, D.; Wieghofer, P.; Staszewski, O.; David, E.; Keren-Shaul, H.; Mahlakoiv, T.; Jakobshagen, K.; Buch, T.; et al. Host microbiota constantly control maturation and function of microglia in the CNS. Nat. Neurosci. 2015, 18, 965-977. [CrossRef]

40. Sampson, T.R.; Debelius, J.W.; Thron, T.; Janssen, S.; Shastri, G.G.; Ilhan, Z.E.; Challis, C.; Schretter, C.E.; Rocha, S.; Gradinaru, V.; et al. Gut Microbiota Regulate Motor Deficits and Neuroinflammation in a Model of Parkinson's Disease. Cell 2016, 167, 1469-1480.e1412. [CrossRef]

41. Chiarini, L.; Bevivino, A.; Dalmastri, C.; Tabacchioni, S.; Visca, P. Burkholderia cepacia complex species: Health hazards and biotechnological potential. Trends Microbiol. 2006, 14, 277-286. [CrossRef] [PubMed]

42. Kaur, H.; Bose, C.; Mande, S.S. Tryptophan Metabolism by Gut Microbiome and Gut-Brain-Axis: An in silico Analysis. Front. Neurosci 2019, 13, 1365. [CrossRef]

43. Heilman, P.L.; Wang, E.W.; Lewis, M.M.; Krzyzanowski, S.; Capan, C.D.; Burmeister, A.R.; Du, G.; Escobar Galvis, M.L.; Brundin, P.; Huang, X.; et al. Tryptophan Metabolites Are Associated With Symptoms and Nigral Pathology in Parkinson's Disease. Mov. Disord. 2020, 35, 2028-2037. [CrossRef] [PubMed]

44. Hsueh, P.-T.; Lin, H.-H.; Liu, C.-L.; Ni, W.-F.; Chen, Y.-L.; Chen, Y.-S. Burkholderia pseudomallei-loaded cells act as a Trojan horse to invade the brain during endotoxemia. Sci. Rep. 2018, 8, 13632. [CrossRef] [PubMed]

45. St John, J.A.; Walkden, H.; Nazareth, L.; Beagley, K.W.; Ulett, G.C.; Batzloff, M.R.; Beacham, I.R.; Ekberg, J.A. Burkholderia pseudomallei Rapidly Infects the Brain Stem and Spinal Cord via the Trigeminal Nerve after Intranasal Inoculation. Infect. Immun. 2016, 84, 2681-2688. [CrossRef]

46. Wallner, A.; King, E.; Ngonkeu, E.L.M.; Moulin, L.; Béna, G. Genomic analyses of Burkholderia cenocepacia reveal multiple species with differential host-adaptation to plants and humans. BMC Genom. 2019, 20, 803. [CrossRef]

47. Kishimoto, Y.; Zhu, W.; Hosoda, W.; Sen, J.M.; Mattson, M.P. Chronic Mild Gut Inflammation Accelerates Brain Neuropathology and Motor Dysfunction in $\alpha$-Synuclein Mutant Mice. Neuromolecular. Med. 2019, 21, 239-249. [CrossRef]

48. Stolzenberg, E.; Berry, D.; Yang, D.; Lee, E.Y.; Kroemer, A.; Kaufman, S.; Wong, G.C.L.; Oppenheim, J.J.; Sen, S.; Fishbein, T.; et al. A Role for Neuronal Alpha-Synuclein in Gastrointestinal Immunity. J. Innate Immun. 2017, 9, 456-463. [CrossRef]

49. Yue, S.; Zhao, D.; Peng, C.; Tan, C.; Wang, Q.; Gong, J. Effects of theabrownin on serum metabolites and gut microbiome in rats with a high-sugar diet. Food Funct. 2019, 10, 7063-7080. [CrossRef]

50. Anderson, G.; Seo, M.; Berk, M.; Carvalho, A.F.; Maes, M. Gut Permeability and Microbiota in Parkinson's Disease: Role of Depression, Tryptophan Catabolites, Oxidative and Nitrosative Stress and Melatonergic Pathways. Curr. Pharm. Des. 2016, 22, 6142-6151. [CrossRef]

51. Duboc, H.; Rajca, S.; Rainteau, D.; Benarous, D.; Maubert, M.-A.; Quervain, E.; Thomas, G.; Barbu, V.; Humbert, L.; Despras, G.; et al. Connecting dysbiosis, bile-acid dysmetabolism and gut inflammation in inflammatory bowel diseases. Gut 2013, 62, 531-539. [CrossRef] [PubMed]

52. Guo, C.; Xie, S.; Chi, Z.; Zhang, J.; Liu, Y.; Zhang, L.; Zheng, M.; Zhang, X.; Xia, D.; Ke, Y.; et al. Bile Acids Control Inflammation and Metabolic Disorder through Inhibition of NLRP3 Inflammasome. Immunity 2016, 45, 802-816. [CrossRef] [PubMed]

53. Ma, C.; Han, M.; Heinrich, B.; Fu, Q.; Zhang, Q.; Sandhu, M.; Agdashian, D.; Terabe, M.; Berzofsky, J.A.; Fako, V.; et al. Gut microbiome-mediated bile acid metabolism regulates liver cancer via NKT cells. Science 2018, 360. [CrossRef] [PubMed] 
54. Praslickova, D.; Torchia, E.C.; Sugiyama, M.G.; Magrane, E.J.; Zwicker, B.L.; Kolodzieyski, L.; Agellon, L.B. The ileal lipid binding protein is required for efficient absorption and transport of bile acids in the distal portion of the murine small intestine. PLoS ONE 2012, 7, e50810. [CrossRef] [PubMed]

55. Mullish, B.H.; McDonald, J.A.K.; Pechlivanis, A.; Allegretti, J.R.; Kao, D.; Barker, G.F.; Kapila, D.; Petrof, E.O.; Joyce, S.A.; Gahan, C.G.M.; et al. Microbial bile salt hydrolases mediate the efficacy of faecal microbiota transplant in the treatment of recurrent Clostridioides difficile infection. Gut 2019, 68, 1791-1800. [CrossRef]

56. Rodrigues, C.M.; Fan, G.; Wong, P.Y.; Kren, B.T.; Steer, C.J. Ursodeoxycholic acid may inhibit deoxycholic acid-induced apoptosis by modulating mitochondrial transmembrane potential and reactive oxygen species production. Mol. Med. 1998, 4, 165-178. [CrossRef]

57. Salen, G.; Colalillo, A.; Verga, D.; Bagan, E.; Tint, G.S.; Shefer, S. Effect of high and low doses of ursodeoxycholic acid on gallstone dissolution in humans. Gastroenterology 1980, 78, 1412-1418. [CrossRef]

58. Mortiboys, H.; Furmston, R.; Bronstad, G.; Aasly, J.; Elliott, C.; Bandmann, O. UDCA exerts beneficial effect on mitochondrial dysfunction in LRRK2(G2019S) carriers and in vivo. Neurology 2015, 85, 846-852. [CrossRef]

59. Sathe, A.G.; Tuite, P.; Chen, C.; Ma, Y.; Chen, W.; Cloyd, J.; Low, W.C.; Steer, C.J.; Lee, B.-Y.; Zhu, X.-H.; et al. Pharmacokinetics, Safety, and Tolerability of Orally Administered Ursodeoxycholic Acid in Patients With Parkinson's Disease-A Pilot Study. J. Clin. Pharmacol. 2020, 60, 744-750. [CrossRef]

60. Dobin, A.; Davis, C.A.; Schlesinger, F.; Drenkow, J.; Zaleski, C.; Jha, S.; Batut, P.; Chaisson, M.; Gingeras, T.R. STAR: Ultrafast universal RNA-seq aligner. Bioinformatics 2013, 29, 15-21. [CrossRef]

61. Segata, N.; Waldron, L.; Ballarini, A.; Narasimhan, V.; Jousson, O.; Huttenhower, C. Metagenomic microbial community profiling using unique clade-specific marker genes. Nat. Methods 2012, 9, 811-814. [CrossRef]

62. Franzosa, E.A.; McIver, L.J.; Rahnavard, G.; Thompson, L.R.; Schirmer, M.; Weingart, G.; Lipson, K.S.; Knight, R.; Caporaso, J.G.; Segata, N.; et al. Species-level functional profiling of metagenomes and metatranscriptomes. Nat. Methods 2018, 15, 962-968. [CrossRef]

63. Paulson, J.N.; Stine, O.C.; Bravo, H.C.; Pop, M. Differential abundance analysis for microbial marker-gene surveys. Nat. Methods 2013, 10, 1200-1202. [CrossRef]

64. Raudvere, U.; Kolberg, L.; Kuzmin, I.; Arak, T.; Adler, P.; Peterson, H.; Vilo, J. g:Profiler: A web server for functional enrichment analysis and conversions of gene lists (2019 update). Nucleic Acids Res. 2019, 47, W191-W198. [CrossRef]

65. Reimand, J.; Isserlin, R.; Voisin, V.; Kucera, M.; Tannus-Lopes, C.; Rostamianfar, A.; Wadi, L.; Meyer, M.; Wong, J.; Xu, C.; et al. Pathway enrichment analysis and visualization of omics data using g:Profiler, GSEA, Cytoscape and EnrichmentMap. Nat. Protoc. 2019, 14, 482-517. [CrossRef]

66. Pan, X.; Elliott, C.T.; McGuinness, B.; Passmore, P.; Kehoe, P.G.; Holscher, C.; McClean, P.L.; Graham, S.F.; Green, B.D. Metabolomic Profiling of Bile Acids in Clinical and Experimental Samples of Alzheimer's Disease. Metabolites 2017, 7, 28. [CrossRef]

67. Ritchie, M.E.; Phipson, B.; Wu, D.; Hu, Y.; Law, C.W.; Shi, W.; Smyth, G.K. Limma powers differential expression analyses for RNA-sequencing and microarray studies. Nucleic Acids Res. 2015, 43, e47. [CrossRef] 